\title{
1 Conservation of function with diversification of higher-order structure within sensor histidine kinases
}

4 Igor Dikiy ${ }^{1}$, Danielle Swingle ${ }^{1,2}$, Kaitlyn Toy ${ }^{1,3}$, Uthama R. Edupuganti ${ }^{1,2}$, Giomar Rivera-Cancel ${ }^{4}$, 5 Kevin H. Gardner ${ }^{1,3,5^{*}}$

$6 \quad$ 1: Structural Biology Initiative, CUNY Advanced Science Research Center, New York, NY 10031

7 2: Biochemistry Ph.D. Program, The Graduate Center - City University of New York, New York, NY 10016

3: Department of Chemistry and Biochemistry, City College of New York, New York, NY 10016

4: Department of Internal Medicine, UT Southwestern Medical Center, Dallas, TX 75390

5: Ph.D. Programs in Biochemistry, Biology, and Chemistry, The Graduate Center - City University of

15 ORCID iDs:

16 Igor Dikiy: 0000-0002-8155-7788

17 Danielle Swingle: 0000-0003-3691-4560

18 Kaitlyn Toy: 0000-0003-2595-8779

19 Uthama R. Edupuganti: 0000-0003-0571-4101

20 Giomar Rivera-Cancel: 0000-0002-4909-4103

21 Kevin H. Gardner: 0000-0002-8671-2556 


\section{Abstract}

Integral to the protein structure/function paradigm, oligomeric state is typically conserved

25 along with function across evolution. However, notable exceptions - such as the hemoglobins -

26 show how evolution can enable new regulatory mechanisms by oligomerization changes. We

27 examine this linkage in the histidine kinases (HKs), widely-distributed prokaryotic environmental

28 sensors. While most HKs are transmembrane homodimers, our finding of a monomeric soluble

$29 \mathrm{HK}$ (EL346, a photosensing Light-Oxygen-Voltage (LOV)-HK) raises questions about the

30 relationship between oligomerization and regulation within this family. Here we characterize

31 multiple EL346 homologs and find a diverse range of HK oligomeric states. Three LOV-HK

32 homologs are dimers with differing structural responses to light, while two are Per-ARNT-Sim

33 (PAS)-HKs that interconvert between differentially-active monomers and dimers, suggesting

34 dimerization as a regulatory control. Finally, we examine putative interfaces in a dimeric LOV-

$35 \mathrm{HK}$, finding that multiple domains contribute to dimerization and function. Our findings suggest

36 potential for novel regulation in this important family of environmental sensors. 


\section{Introduction}

Sensor histidine kinases (HKs) are signal transduction receptors that are widespread in prokaryotic organisms, enabling them to sense and respond to diverse environmental stimuli (Galperin, 2018). HKs are typically found in two-component systems (TCS), which are most

42 simply composed of a sensor HK and a downstream response regulator (RR) collocated in the

43 same operon. The RR responds to a signal from the HK by acting as a phosphorylation-

44 dependent transcription regulator, usually binding to DNA and controlling gene expression in

45 various bacterial processes, including virulence (Kenney \& Anand, 2020; Mechaly et al., 2017;

46 Wang, 2012; West \& Stock, 2001). The majority of sensor HKs characterized to date are

47 obligate homodimeric transmembrane receptors, facilitating transmission of signals across the

48 membrane from periplasmic sensor domains to cytoplasmic kinase domains via intermonomer, 49 symmetry-breaking conformational changes (Bhate et al., 2015; Gushchin \& Gordeliy, 2018;

50 Szurmant et al., 2007). In contrast, some sensor HKs adopt varying oligomeric states, ranging

51 from large multimeric assemblies observed for bacterial chemosensors (Muok et al., 2020) to

52 the monomeric soluble HK EL346 (Rivera-Cancel et al., 2014). Soluble sensor HKs are relieved

53 of the constraints of transmitting a signal across a lipid bilayer, which typically necessitates

54 signal transmission via motions of one monomer with respect to the other (Bhate et al., 2015;

55 Herrou et al., 2017). This raises the open question: Are such soluble HKs more likely to adopt

56 varied oligomeric states including non-dimeric architectures? We view the relatively

57 understudied HWE/HisKA2 family of HKs (Herrou et al., 2017), which is enriched in soluble

58 cytoplasmic proteins that participate in atypical signaling pathways, to be a tempting testbed for

59 this hypothesis.

Typical sensor HKs contain an $\mathrm{N}$-terminal sensor domain and a C-terminal kinase

61 domain consisting of a two-helix dimerization histidine phosphotransfer (DHp) domain and a

62 catalytic ATP-binding (CA) domain (Bhate et al., 2015). Stimulus detection at the sensor

63 domain, or in some cases at other domains (Kenney \& Anand, 2020), modulates the CA domain

64 autophosphorylation of a conserved His residue in the $\mathrm{DHp}$ domain, a signal which is

65 subsequently transferred to a conserved Asp in the RR receiver domain (West \& Stock, 2001).

66 The homodimeric nature of canonical transmembrane HKs is largely believed to be required for

67 both the structural changes between the "off" and "on" state and determining whether

68 autophosphorylation occurs between monomers (in trans) or in cis within a monomer

69 (Ashenberg et al., 2013; Bhate et al., 2015). Dimerization is mediated via the DHp domains of

70 two monomers, forming a four-helix bundle and facilitating pivot-, piston-, or scissoring-type 
71 movements as part of the activation process (Bhate et al., 2015; Herrou et al., 2017), echoing

72 similar themes in integrins (Luo et al., 2007) and certain other transmembrane receptors.

This traditional view of sensor HK structure has been built up from extensive structural and biochemical studies (Bhate et al., 2015), most of which have focused on HKs in only one of several subfamilies of histidine kinases (Pfam (El-Gebali et al., 2019) family HisKA). In contrast, the related HWE-HK and HisKA_2 families (referred to here as the HWE/HisKA2 family) of HKs have not been as extensively studied from a structural perspective (Herrou et al., 2017). This family, which differs from the canonical HisKA family in the DHp and CA domains, appears to be enriched in HKs lacking transmembrane segments and correspondingly likely localized to the cytosol. These HKs also tend to signal in an atypical manner, utilizing phosphorylation to control protein-protein interactions of RRs, rather than directly impacting them as transcription factors per se (Herrou et al., 2017). HWE/HisKA2 HKs are also enriched in the Alphaproteobacteria (Karniol \& Vierstra, 2004; Staroń \& Mascher, 2010).

Our prior work on the HWE/HisKA2 family has focused on three soluble HKs involved in the general stress response of the marine alphaproteobacterium Erythrobacter litoralis HTCC2594 (EL346, EL362, and EL368) (Correa et al., 2013; Rivera-Cancel et al., 2014; Swartz et al., 2007). Each of these proteins senses blue light through a light oxygen voltage (LOV) sensor domain (Crosson et al., 2003; Pudasaini et al., 2015), a sub-class of the versatile PerARNT-Sim (PAS) domains that are present in over 30\% of HKs (Gao \& Stock, 2009). The response to blue light provides an experimentally tractable way to explore the inactive and active states of histidine kinases, typically corresponding to dark and light states of LOV-HKs (Möglich, 2019; Swartz et al., 2007; Tseng et al., 2010). These states can be conveniently distinguished spectrophotometrically: while the dark state spectra of LOV-HKs exhibit a triplet peak at around $450 \mathrm{~nm}$, characteristic of protein-bound flavin chromophores, the light state spectrum has a broad, singlet absorption peak at $390 \mathrm{~nm}$ (Zoltowski \& Gardner, 2011). This spectroscopic change is due to the formation of a photochemically-generated covalent adduct between the $\mathrm{C}(4 \mathrm{a})$ of the isoalloxazine ring and a conserved cysteine residue, a bond that is thermally reversible in the dark on a timescale varying from seconds to hours among LOV domains (Pudasaini et al., 2015).

While our prior studies indicate that EL368 and EL362 (when reconstituted with flavin) are dimeric, EL346 is a soluble and functional monomer (Correa et al., 2013; Rivera-Cancel et

102 al., 2014). The EL346 crystal structure reveals that it is held in a monomeric state by an

103 intramolecular association between its DHp-like domain and the LOV sensory domain, blocking 104 the face typically used for DHp domains to dimerize (Rivera-Cancel et al., 2014). Despite this 
substantial deviation from current HK signaling models, the monomeric EL346 fully responds to stimulus with a light-induced increase in autophosphorylation (Rivera-Cancel et al., 2014). More broadly, EL346 suggests that soluble HKs may not require dimerization as a key element in signal transduction, unlike conventional transmembrane HKs. Thus, we set out to characterize the oligomeric and functional states of several novel HWE/HisKA2 HKs.

To do so, we used sequence homology searches to identify multiple soluble LOV- and PAS-HKs with similarity to EL346 and used a combination of approaches to test if these proteins were similarly functional monomers. We report here our characterization of three novel LOV-HKs, each of which properly photocycle by UV-visible absorbance spectroscopy and are dimeric with a range of light-triggered conformational changes, as detected by size exclusion chromatography coupled with multi-angle light scattering (SEC-MALS). In contrast, we also detail results from two light-insensitive PAS-HKs which exist in monomeric and dimeric states with differing autophosphorylation activity, suggesting dimerization as a possible regulatory switch in this family of HKs. Finally, we combine structural and sequence information to engineer a "monomerized dimer" by deleting putative dimerization interfaces in a dimeric LOV$\mathrm{HK}$, finding that a distributed interface of interactions encodes both dimerization and autokinase activity. Taken together, our data indicate that a wider range of oligomerization states are accessible to HKs, raising exciting questions about regulation and evolution potentially analogous to other protein families which exhibit differences in quaternary structure and control, such as the hemoglobins (Pillai et al., 2020).

\section{Methods}

Bioinformatics

EL346 homologs were identified by searching for non-redundant protein sequences in the GenBank database using BLAST with EL346 amino acids 1-346 as query. EL346 and the best 100 sequences were aligned using Clustal Omega (Sievers et al., 2011) and a distance tree was calculated from this alignment using the same software. Graphic display of this tree was accomplished using iTOL (Interactive Tree of Life, http://itol.embl.de/) (Letunic \& Bork, 2019) and Adobe Illustrator CS6. This tree was used to select HKs for cloning from the strains available at DSMZ (http://www.dsmz.de).

\section{Cloning, protein expression and purification}

The HKs selected from each strain are shown in Table 1. The proteins were cloned into a pHis-G $\beta 1$ vector (Sheffield et al., 1999). Constructs were verified by DNA sequencing. The 
proteins were overexpressed in Escherichia coli BL21(DE3) (Stratagene) in LB as previously described (Dikiy et al., 2019). Cells were harvested, resuspended in Buffer A (50 mM Tris pH 8.0, $100 \mathrm{mM} \mathrm{NaCl}$, and $10 \mathrm{mM} \mathrm{MgCl}_{2}$ ), and lysed by sonication. The lysate was centrifuged at

$14248,000 \times \mathrm{g}$ and $4{ }^{\circ} \mathrm{C}$ for $45 \mathrm{~min}$. The supernatant was loaded into a $\mathrm{Ni}^{2+}$ Sepharose affinity

143 column (GE Healthcare) and the His6-G $\beta 1$ tagged protein was washed with 4 column volumes

144 of Buffer A supplemented with $15 \mathrm{mM}$ imidazole and eluted with 4 column volumes of Buffer $\mathrm{A}$

145 supplemented with $250 \mathrm{mM}$ imidazole. Eluted proteins were exchanged into Buffer A by dialysis.

146 The fusion tag was cleaved by incubation with His6-TEV protease overnight at $4{ }^{\circ} \mathrm{C}$. The tagless proteins were separated from the tags and His6-TEV protease by $\mathrm{Ni}^{2+}$ affinity chromatography and were further purified by size-exclusion chromatography on a HiLoad 16/600 Superdex 200 column (GE Healthcare) equilibrated with HSEC buffer containing 50 mM HEPES pH 7.5, $100 \mathrm{mM} \mathrm{NaCl}, 10 \mathrm{mM} \mathrm{MgCl}_{2}$, and $0.5 \mathrm{mM} \mathrm{DTT}$. For light-sensitive proteins, all purification steps were performed under dim red light. Concentrations were determined from the

152 theoretical absorption coefficient, $\varepsilon_{280}$ for PAS-HKs, calculated from the sequence using the

153 ExPASy ProtParam server (Gasteiger et al., 2005), and $\varepsilon_{446}=11,800 \mathrm{M}^{-1} \mathrm{~cm}^{-1}$ for flavin-

154 containing proteins.

155 RH376 deletion mutants were generated as follows: $\mathrm{RH} 2$ and $\mathrm{RH} 3$ were ordered as

156 synthetic constructs from Genewiz (South Plainfield, NJ) and inserted into the same pHis-G $\beta 1$

157 vector as the WT protein. The RH1 deletion was generated by PCR using an N-terminal primer

158 lacking the sequence to be deleted using WT and $\mathrm{RH} 3$ plasmids as templates, to generate $\mathrm{RH} 1$

159 and $\mathrm{RH} 1+3$, respectively.

Absorbance and dark state reversion kinetics

UV-visible absorbance spectroscopy measurements were acquired using a Varian Cary

16350 spectrophotometer at $24{ }^{\circ} \mathrm{C}$ with a $1 \mathrm{~cm}$ path length quartz cuvette. First, a UV-visible

164 spectrum in the range $250-550 \mathrm{~nm}$ was acquired in light and dark conditions for each LOV-HK.

165 For dark measurements, all steps were performed under dim red light. For light measurements, 166 the samples were illuminated for 1 minute using a blue LED panel (225 LED bulbs in $30.5 \times$

167 30.5-cm panel, $13.8 \mathrm{~W}, 465 \mathrm{~nm}$ maximum illumination wavelength; LEDwholesalers [Hayward,

$168 \mathrm{CA}]$ ) prior to each scan. To measure dark state recovery, each LOV-HK sample was illuminated 169 under a blue LED panel for 1 minute and then transferred to the spectrophotometer. $A_{446}$ of the 170 samples in HSEC buffer with $1 \mathrm{mM}$ ATP was monitored at 5-min intervals for 400-800 min at

$17124^{\circ} \mathrm{C}$. Time constants $\tau$ were determined by fitting measurements to a monoexponential decay

172 using the following equation: 
Abs $=A_{\text {inf }}-\left(A_{\text {inf }}-A_{o}\right) e^{-t / \tau}$, where $A_{\text {inf }}=A_{446}$ at infinity, $A_{o}=A_{446}$ initial, and $t=$ time

174 Raw data were fit and plotted using RStudio and plots were edited in Adobe Illustrator CS6.

SEC-MALS

All samples and buffers were filtered with a 0.1-um pore size filter before use. ATP was

178 added to each $\mathrm{HK}$ sample $(\sim 15 \mu \mathrm{M}, 400 \mu \mathrm{L})$ to a final concentration of $1 \mathrm{mM}$. Samples were

179 then injected at a $0.4 \mathrm{~mL} / \mathrm{min}$ flow rate onto a Superdex $200 \mathrm{GL}$ 10/300 SEC column (GE

180 Healthcare) using HSEC buffer with $1 \mathrm{mM}$ ATP. For lit experiments, samples were illuminated with a blue LED panel for 1 min prior to injection and the blue LED panel was faced toward the column during the run. For dark experiments, all steps were performed under dim red light. Samples were detected post-elution by the inline miniDAWN TREOS light scattering and Optilab rEX refractive index detectors (Wyatt Technology [Santa Barbara, CA]). FPLC runs were performed at $4{ }^{\circ} \mathrm{C}$, while MALS and refractive index measurements were at $25^{\circ} \mathrm{C}$. Data analysis and molecular weight calculations were performed using the ASTRA V software (Wyatt Technology). Raw data were plotted using RStudio and plots were edited in Adobe Illustrator CS6 and overlaid in Adobe Photoshop CS6.

Autophosphorylation assays

Autophosphorylation was measured as described previously (Dikiy et al., 2019; Rivera-

192 Cancel et al., 2014). Two trials were conducted for each protein under each condition. Proteins 193 were added to a reaction buffer containing $50 \mathrm{mM}$ Tris, $100 \mathrm{mM} \mathrm{NaCl}, 5 \mathrm{mM} \mathrm{MnCl}$, and $1 \mathrm{mM}$

194 DTT with $\mathrm{pH}$ 8.2. Protein concentrations were confirmed by analysis of dilution series on 195 Coomassie-stained SDS-PAGE. Concentrations typically ranged from 4.7 to $19.8 \mu \mathrm{M}$, with

196 EL346 and RH2 able to be more concentrated at 34.1 and $67.1 \mu \mathrm{M}$. A mixture of unlabeled ATP 197 and $10 \mu \mathrm{Ci}\left[\gamma_{-}{ }^{32} \mathrm{P}\right]$ ATP was added to each mixture to initiate the reaction (final ATP 198 concentration of $3.6 \mu \mathrm{M})$. Aliquots were removed at time points of $0.5,1,2,4,8$, 6, and $32 \mathrm{~min}$ 199 and placed into a 4x SDS-gel loading buffer to quench the reaction. For dark measurements, all 200 steps were performed under dim red light. For light measurements, the samples were 201 illuminated with a blue LED panel just prior to and throughout the course of the experiment.

202 Samples were subjected to SDS-PAGE analysis; dried gels were exposed overnight and bands 203 were visualized by phosphorimaging. Band intensities were measured using Fiji (Rueden et al., 204 2017; Schindelin et al., 2012); initial velocity values were calculated in Microsoft Excel from the linear region of the curve and were plotted in RStudio and edited in Adobe Illustrator CS6. 


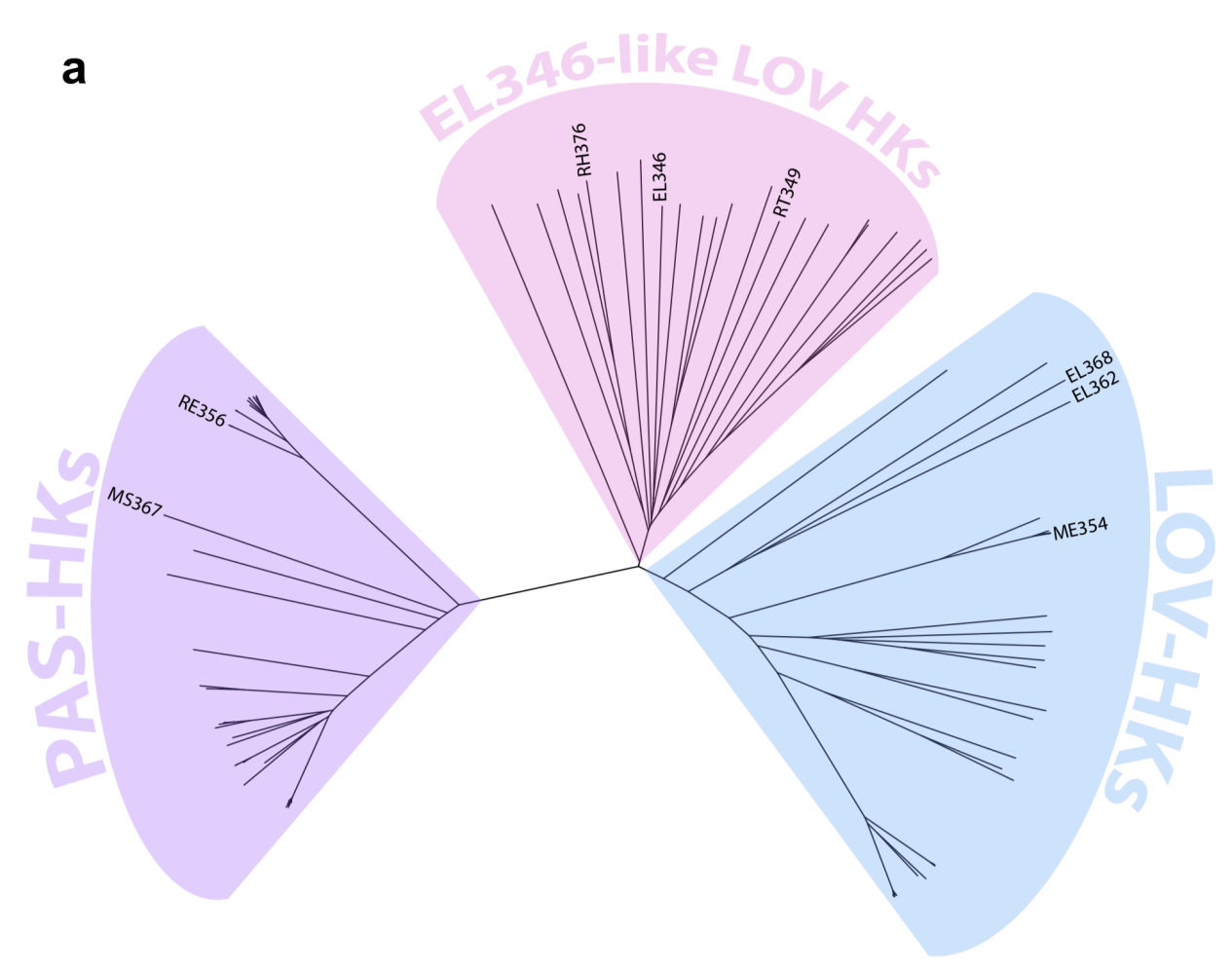

b
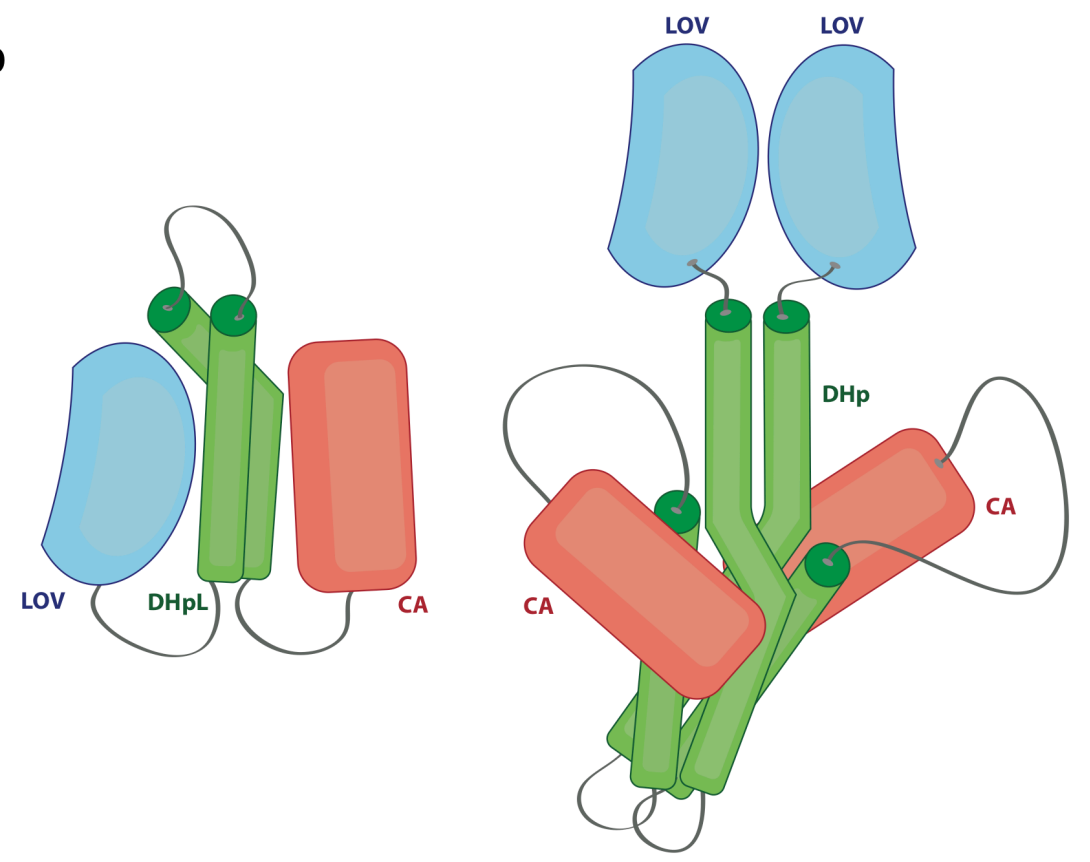

Figure 1: EL346 homologs form three distinct clades. a) PAS-HKs (purple), EL346-like LOV HKs (pink), and LOV-HKs (blue). The names of the proteins from each clade that were selected for this study are shown. All other names are omitted for simplicity and can be found in Figure S2. b) Schematic structures of a monomeric (left) and dimeric (right) LOV-HK. Monomeric schematic is based on the structure of EL346 (Rivera-Cancel et al., 2014) and dimeric is based on the fusion protein YF1 (Diensthuber et al., 2013). 


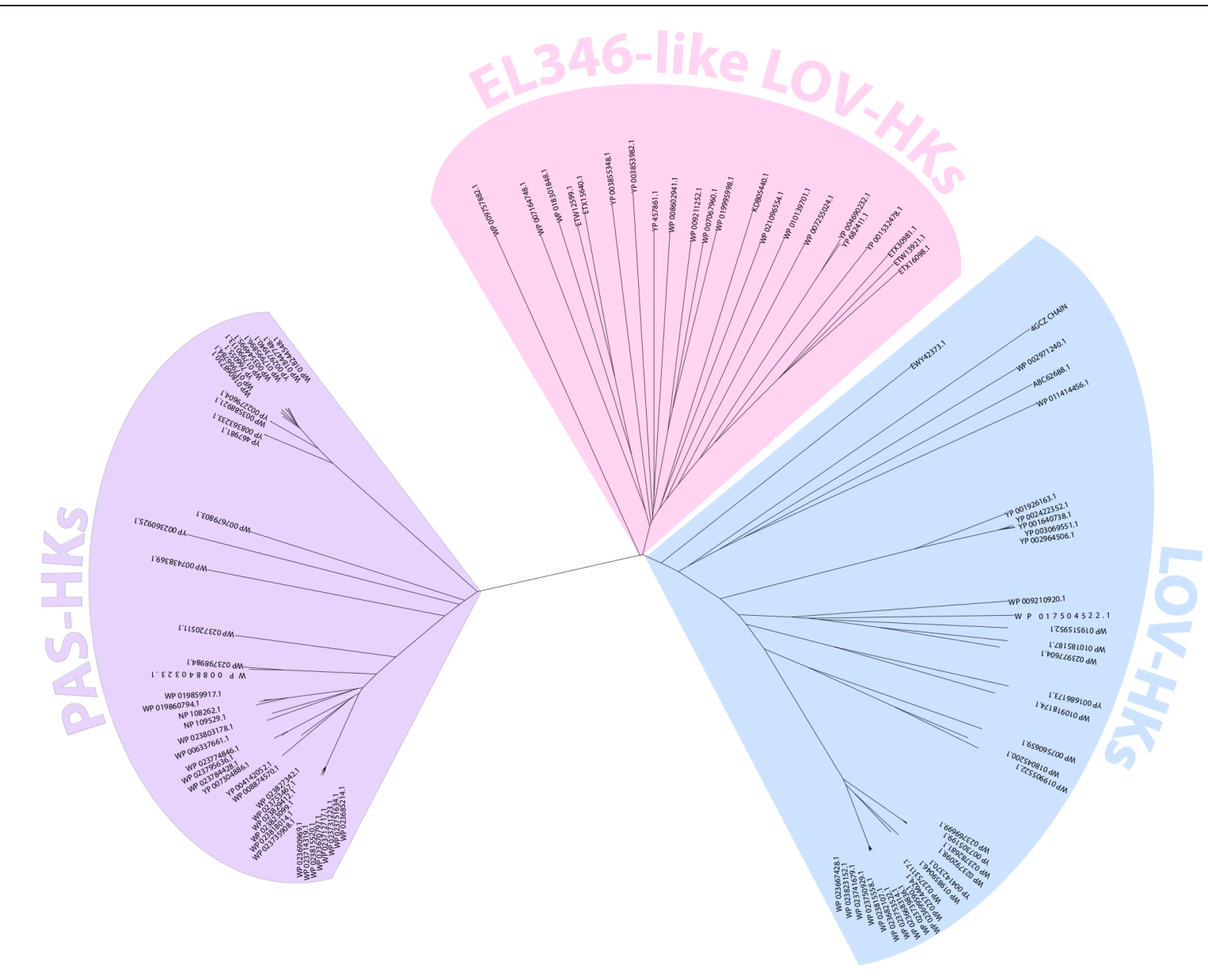

Figure S2: MSA Tree of EL346 Homologs (Supplemental to Fig. 1). NCBI reference numbers for each of the 100 EL346 homologues shown in Figure 1 are listed here.

\section{Results}

210 Bioinformatics analysis reveals numerous alphaproteobacterial EL346 homologs

211 To identify potential additional monomeric histidine kinases, we searched for homologs

212 to the full-length EL346 sequence as a well-validated, functional monomer. Taking the top 100

213 BLASTp hits (in addition to EL346), all belonging to the Alphaproteobacteria, we generated a

214 multiple sequence alignment of proteins which all contained the same domain architecture of a

215 single N-terminal PAS or LOV domain followed by a C-terminal HWE/HisKA2 histidine kinase,

216 and without any predicted transmembrane segments. A distance tree analysis revealed three

217 clades (Fig. 1a) which split on sensor type: PAS-HKs (non-LOV), LOV-HKs, and EL346-like

218 LOV-HKs. Interestingly, a subgroup of the EL346-like LOV-HKs is missing the conserved

219 phosphoacceptor histidine (Fig. S1). We also included the related EL368 (dimer) and EL362

220 (blind monomer) histidine kinases from E. litoralis HTCC2594 that we had previously identified 
221 (Correa et al., 2013), as well as the Caulobacter crescentus LovK (dimer) (Purcell et al., 2010)

222 in the alignment; all three of these proteins clustered in the LOV-HKs clade. Additionally, our

223 search identified a third PAS-HK clade with proteins containing non-LOV PAS domains with 17-

$22424 \%$ sequence identity to the EL346 LOV domain. Notably, many of these domains contained a

225 conserved tryptophan in place of the cysteine utilized in LOV-type photochemistry (Crosson \&

226 Moffat, 2001) (Fig. S1). These data show that the PAS/LOV-HK architecture is widespread

227 throughout Alphaproteobacteria and contains members which could well respond to stimuli

228 other than blue light.

\begin{tabular}{|c|c|c|c|c|c|c|}
\hline \multirow{4}{*}{ 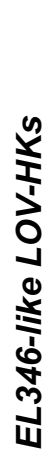 } & Organism & $\begin{array}{l}\text { Protein } \\
\text { Name }\end{array}$ & $\begin{array}{l}\text { Uniprot } \\
\text { ID }\end{array}$ & NCBI ref. & $\begin{array}{l}\text { Identity to } \\
\text { EL346 }\end{array}$ & $\begin{array}{l}\text { Oligomeric } \\
\text { State }\end{array}$ \\
\hline & $\begin{array}{c}\text { Erythrobacter } \\
\text { litoralis HTCC2594 }\end{array}$ & EL346 & Q2NB77 & YP_457861.1 & $\mathrm{N} / \mathrm{A}$ & Monomer \\
\hline & $\begin{array}{l}\text { Rubellimicrobium } \\
\text { thermophilum } \\
\text { DSM } 16684\end{array}$ & RT349 & S9SB69 & WP_021096554.1 & $42.3 \%$ & Dimer \\
\hline & $\begin{array}{c}\text { Roseivivax } \\
\text { halodurans JCM } \\
10272\end{array}$ & $\mathrm{RH} 376$ & X7EIB8 & ETX15640.1 & $35.8 \%$ & Dimer \\
\hline \multirow{3}{*}{$\frac{n}{\frac{n}{5}}$} & $\begin{array}{l}\text { Methylorubrum } \\
\text { extorquens DM4 }\end{array}$ & ME354 & C7CCX6 & YP_003069551.1 & $29.6 \%$ & Dimer \\
\hline & $\begin{array}{c}\text { Erythrobacter } \\
\text { litoralis HTCC2594 }\end{array}$ & EL368 & Q4TL45 & ABC62688.1 & $25.5 \%$ & Dimer \\
\hline & $\begin{array}{c}\text { Erythrobacter } \\
\text { litoralis HTCC2594 }\end{array}$ & EL362 & Q2N9L9 & WP_011414456.1 & $25.8 \%$ & Monomer \\
\hline \multirow{2}{*}{$\frac{y}{\frac{y}{x}}$} & $\begin{array}{l}\text { Rhizobium etli } \\
\text { CFM } 42\end{array}$ & RE356 & Q2KD32 & YP_4679981.1 & $30.1 \%$ & $\begin{array}{l}\text { Monomer/ } \\
\text { Dimer }\end{array}$ \\
\hline & $\begin{array}{l}\text { Methylocella } \\
\text { silvestris BL2 }\end{array}$ & MS367 & B8ELH4 & YP_002360925.1 & $28.7 \%$ & $\begin{array}{l}\text { Monomer/ } \\
\text { Dimer }\end{array}$ \\
\hline 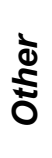 & $\begin{array}{c}\text { Bacillus subtilis/ } \\
\text { Bradyrhizobium } \\
\text { japonicum }\end{array}$ & $\begin{array}{c}\text { YF1 } \\
\text { (engineered } \\
\text { YtvA/FixL } \\
\text { fusion) }\end{array}$ & $\mathrm{N} / \mathrm{A}$ & N/A & $25.5 \%$ & Dimer \\
\hline
\end{tabular}

Table 1: Oligomeric states of known LOV-HKs and PAS-HKs include mix of monomers and dimers. Soluble HKs shown here are separated by clade with the same coloring as Fig. 1 (EL346-like LOV HKs - pink, LOV-HKs - blue, PAS-HKs - purple, Other - grey) and include those investigated in this study (EL346, RT349, RH376, ME354, RE356, and MS367) as well as those identified in literature (EL368, EL362, and YF1) (Correa et al., 2013; Diensthuber et al., 2013). Oligomeric states were determined in this study by SEC-MALS. 
231 LOV-HK homologs are light-sensing and appear mostly dimeric

232 We posited that the EL346-like LOV-HK clade might contain other monomeric HKs

233 similar to EL346, in addition to other members which might adopt the more commonly-observed

234 dimeric assembly seen in the engineered LOV-HK YF1 (Diensthuber et al., 2013), as illustrated

235 in Fig. 1b. To test this hypothesis, we selected several HKs from each clade and characterized

236 their oligomeric state and function with a variety of biochemical assays. We started by

237 determining the ability of the LOV-containing proteins to bind flavin chromophores and undergo

238 typical LOV photochemistry via UV-visible absorbance spectroscopy. The dark and light spectra

239 of four representative proteins, EL346, ME354, RT349, and RH376 (see Table 1 for protein

240 details) all show the characteristic flavin-LOV triplet $(\sim 50 \mathrm{~nm})$ in the dark state which

241 disappears upon illumination (Fig. 2a). Upon incubation in the dark, the $450 \mathrm{~nm}$ absorbance

242 returns with exponential kinetics and a time constant that varies by protein: the other LOV-HKs

243 measured here, such as ME354 (236 min), RT349 (240 min), and RH376 (62 min), had longer

244 reversion constants $\tau$ than EL346 (33 $\mathrm{min}$ ) in the presence of $1 \mathrm{mM}$ ATP (Fig. 2b). The notably

245 long reversion time constants of ME354 and RT349 can be explained by the presence of

246 several "slowing" mutations in their LOV domains relative to that of EL346, specifically at

247 positions 19, 21, 32, and 101 (EL346 numbering), as collated in a review by Pudasaini and

248 coworkers (Pudasaini et al., 2015).

249 We determined the oligomeric state of these LOV-HKs in the presence of ATP and either

250 dark or light state, using size exclusion chromatography coupled to multi-angle light scattering

251 (SEC-MALS), starting by confirming that EL346 is monomeric under both conditions with

252 minimal change in shape upon illumination as revealed by SEC elution profiles (Fig. 2c). In

253 contrast, the three other LOV-HK proteins are stable dimers (via MALS data) but undergo a

254 range of light-dependent shape changes which impact SEC elution times. These range from

255 minimal, with ME354 eluting as a single peak that does not noticeably change between dark

256 and light, to substantial shifts to later elution times for both RT349 and RH376 consistent with a

257 light-induced compaction. Overall, our results suggest that other LOV-HKs are generally dimeric

258 and exhibit varying global conformational changes in response to light.

260 Non-LOV PAS-HKs show mix of monomers and dimers, both of which are active

The two PAS-HKs we characterized, from Rhizobium etli CFN 42 (RE356) and from

262 Methylocella silvestris BL2 (MS367), appear as a mixture of dimer and monomer, as well as an

263 oligomeric or aggregated fraction, as confirmed by SEC-MALS (Fig. 3a). These two proteins

264 exhibit $45 \%$ sequence identity to one another (32\% over the PAS domain) and behave very 


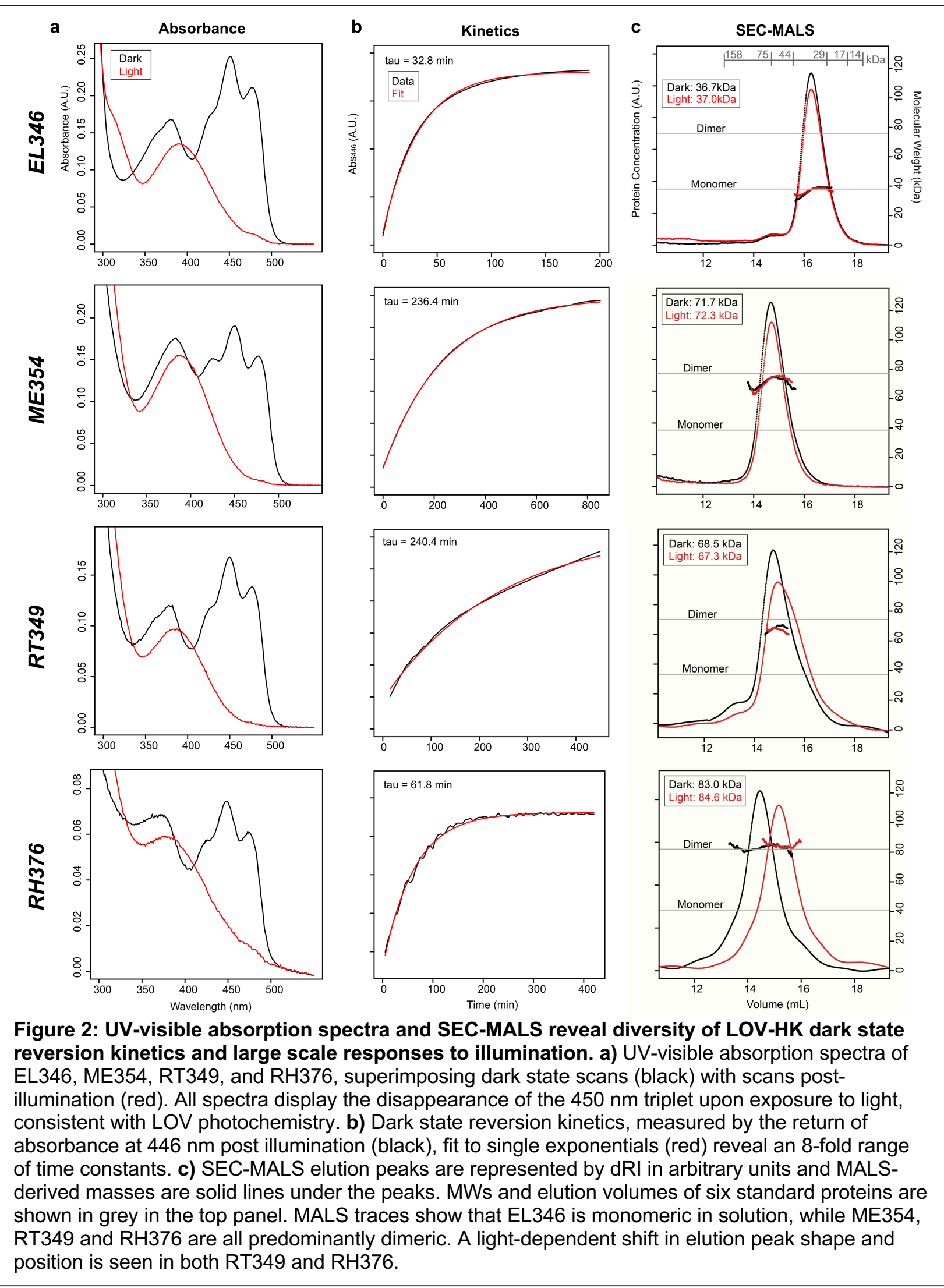


a
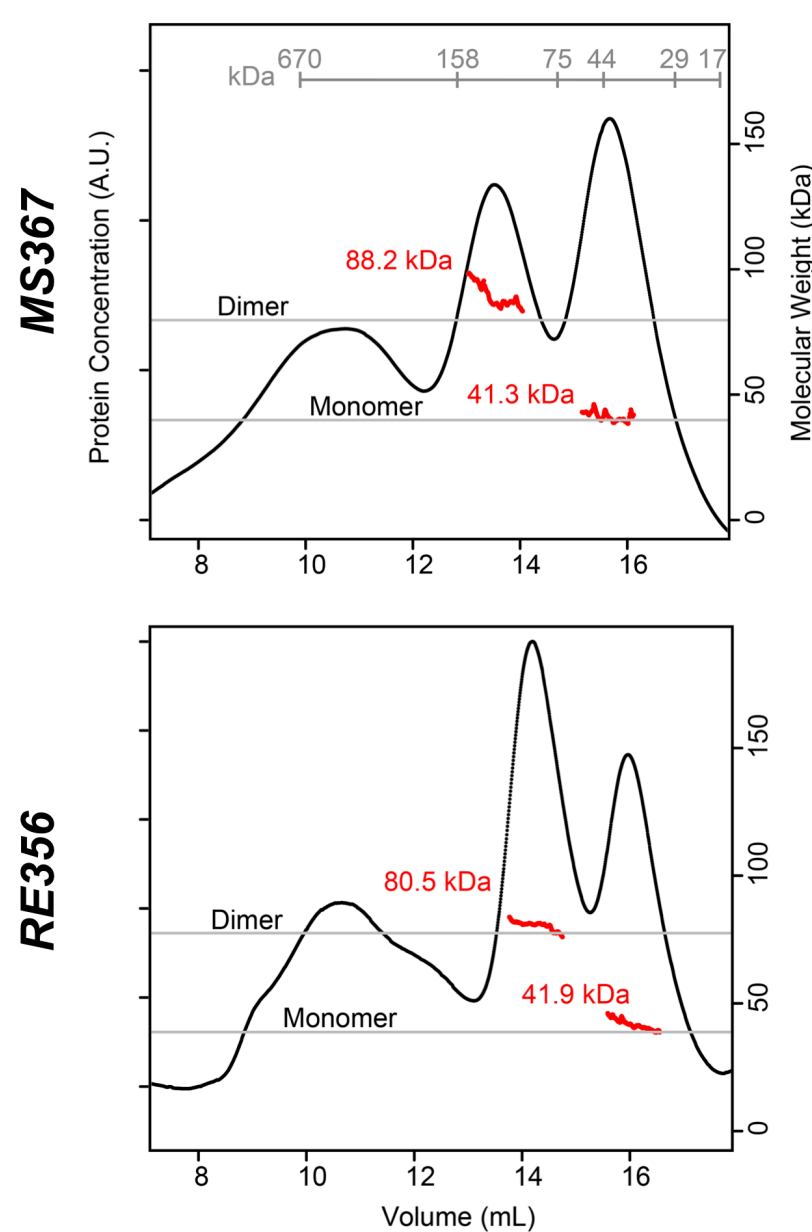

b Autophosphorylation Activity
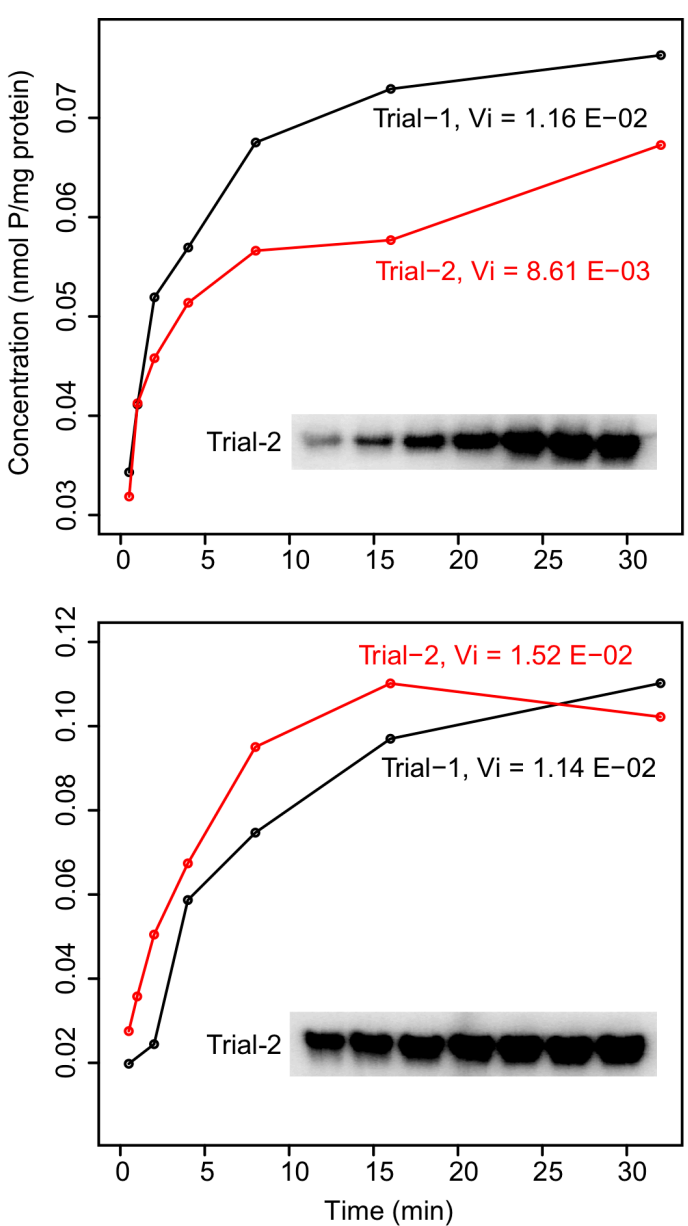

Figure 3: The MS367 and RE356 PAS-HKs are monomer/dimer mixtures with autophosphorylation activities. a) SEC-MALS measurements of PAS-HKs MS367 and RE356 reveal the presence of both monomeric and dimeric states (mass, red traces). The elution peaks (black trace) of each protein are consistent with the expected volumes, suggesting both adopt globular states. b) Autophosphorylation activity curves suggest that both PAS-HKs are active. Two trials were conducted for each protein (mixture of monomer/dimer) and both measurements are shown here. One representative gel is shown for each protein. Initial velocities for each trial were calculated from the linear region of these curves.

similarly in our biophysical tests and activity assays. While these proteins lack a known trigger analogous to light for the LOV-HKs, we still wanted to determine whether both proteins exhibited any autophosphorylation in their default state as purified from E. coli. Both MS367 and RE356

271 displayed autophosphorylation activity approximately one order of magnitude higher than that of

272 EL346 as determined previously (Dikiy et al., 2019; Rivera-Cancel et al., 2014) and confirmed in

273 this study (see below), with the characteristic plateau indicating an equilibrium between kinase

274 and phosphatase activity (Fig. 3b). The activity of RE356 was slightly higher than that of

275 MS367, with approximately 1.5-fold greater initial velocity. It is unclear whether this level of 
activity indicates a high basal activity or whether binding to the putative trigger turns off the

277 kinase.

We wondered whether oligomeric state (monomer vs dimer) affects autophosphorylation activity. We found that the monomeric and dimeric states of MS367 and RE356 are in slow equilibrium, and could be separately injected onto SEC-MALS, as shown for MS367 in Fig. $\mathbf{4 a .}$ We also determined autophosphorylation activity separately for the monomer and dimer

282 fractions of MS367 and found that the dimer is approximately 4-fold more active than the 283 monomer fraction (Fig. 4b). However, the dimeric fraction also exhibited a large proportion of

284 oligomer/aggregate, suggesting that the dimeric state is less stable or prone to further oligomerization (Fig. 4a). Using size-exclusion chromatography, we determined that the monomeric state of RE356 is stable on the order of days at $4{ }^{\circ} \mathrm{C}$, while the dimer slowly oligomerizes or aggregates on this timescale (Fig. 4c). These data illustrate that active monomeric HKs are to be found in this family, but dimerization may serve as a regulatory mechanism.

\section{a MS367 Stability}
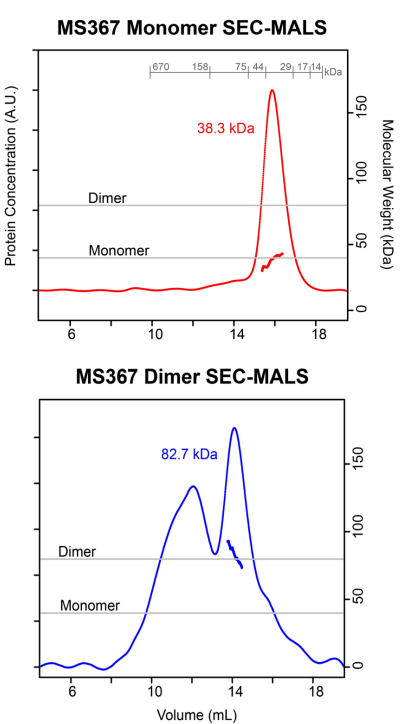

b MS367 Activity

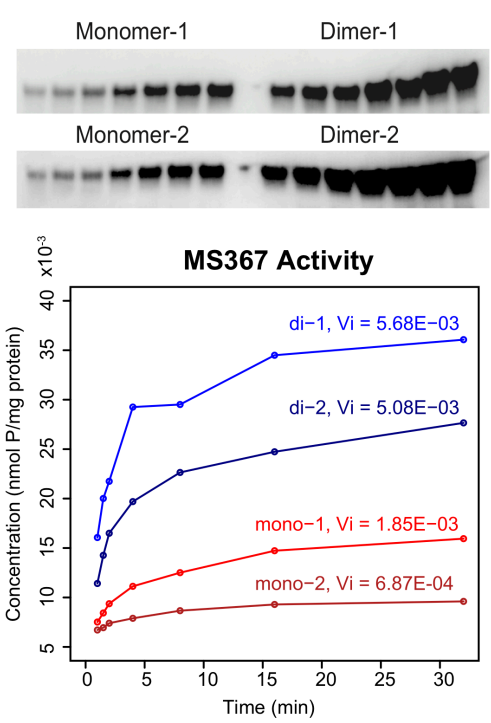

C RE356 Stability

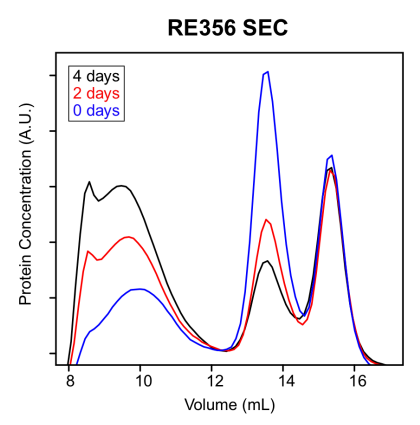

Figure 4: PAS-HK monomer/dimer states can be separated, dimer is more active than monomer, and monomer is more stable over time. a) MS367 monomers and dimers were separated and injected onto a Superdex 200 GL 10/300 SEC column. The resulting MALS-derived masses (solid lines under elution peaks) show that both remain stable upon separation. b) MS367 autophosphorylation assays (phosphor images, top and plots, bottom) of the monomer (red traces) and dimer (blue traces) of MS367 show that the dimer has more autophosphorylation activity. c) SEC traces of RE356 dimer and monomer after 0,2 , and 4 days at $4{ }^{\circ} \mathrm{C}$ are shown, revealing that the monomer is stable over time, while the dimer tends to aggregate. 
bioRxiv preprint doi: https://doi.org/10.1101/2020.07.08.194225; this version posted July 10, 2020. The copyright holder for this preprint (which was not certified by peer review) is the author/funder, who has granted bioRxiv a license to display the preprint in perpetuity. It is made available under aCC-BY 4.0 International license.

RH376 deletion mutants: RH1 RH2 RH3 RH4

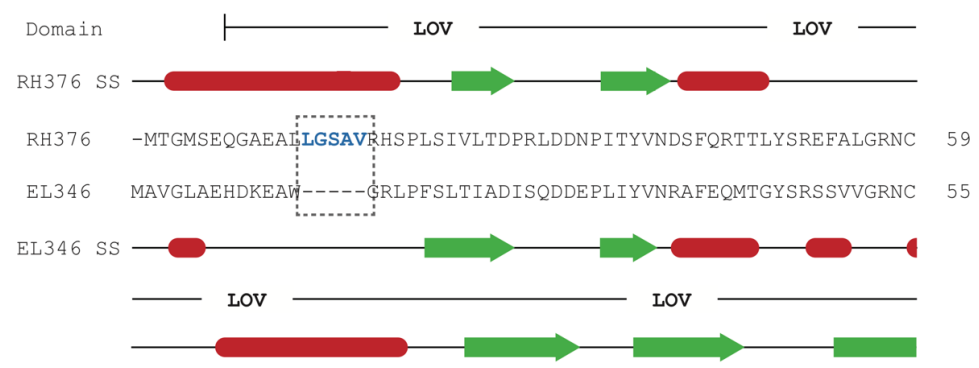

RH376 RFLQGEETDPRDVQRMRDGLTAREQFELTIVNHKADGTPFTNQLLISPVHDDDGEVVAFF 119

EL346 RFLQGEKTDPGAVERLAKAIRNCEEVEETIYNYRADGEGFWNHLLMGPLEDQDEKCRYFV 115

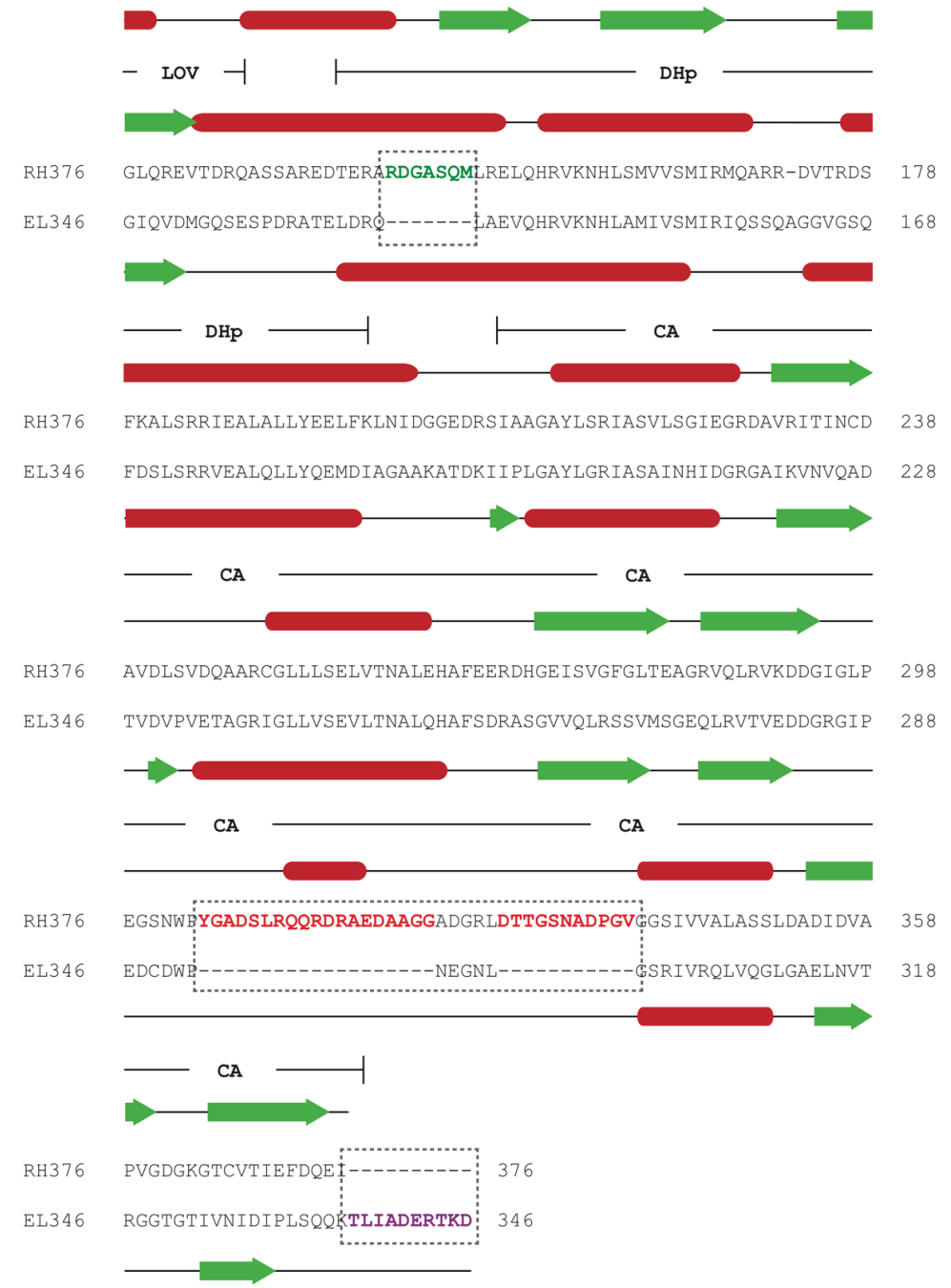

Figure S3: Pairwise alignment of EL346 and RH376 reveals "gaps" in EL346 sequence (Supplemental to Fig. 5). The pairwise alignment of EL346 and RH376 sequences was performed by MUSCLE (Edgar, 2004). "Insertion" regions of RH376 termed RH1-4 are highlighted with dashed boxes. Secondary structure prediction by JPred (Drozdetskiy et al., 2015) (red = helices, green = sheets) for RH376 and from the 4R3A PDB entry for EL346 (Rivera-Cancel et al., 2014) as well as domain locations (LOV, DHp, and CA) are shown next to the amino acid sequences. 


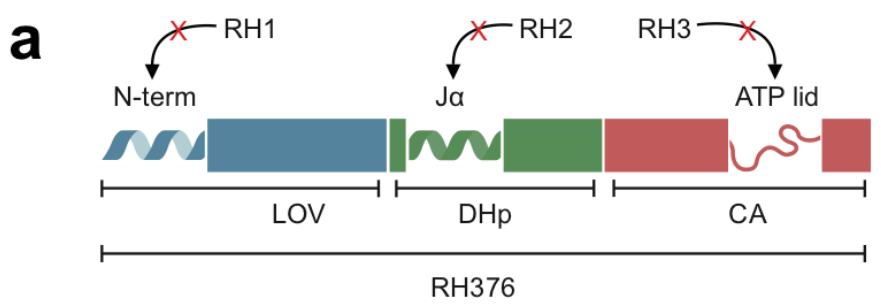

b

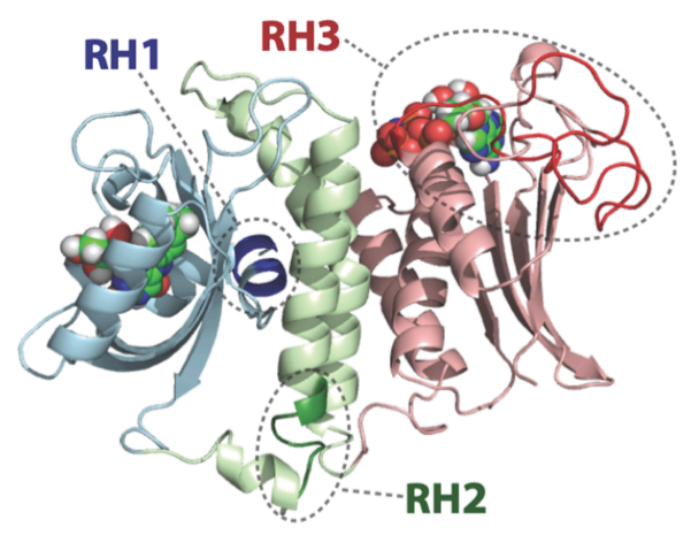

C

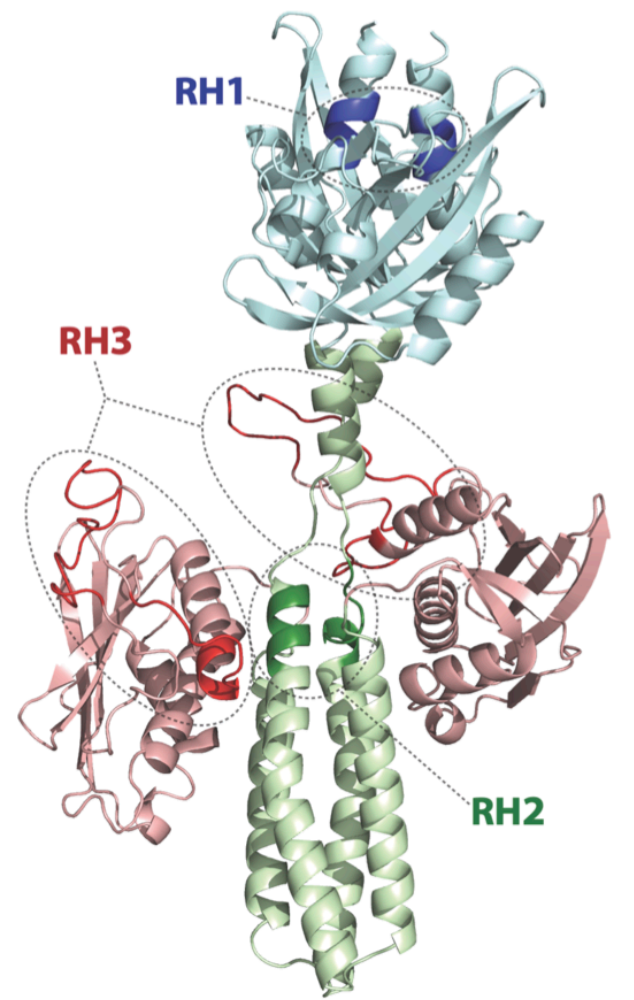

Figure 5: RH376 mutant deletion regions are designed in predicted dimer interfaces. $\mathrm{RH} 376$ deletion mutants $\mathrm{RH} 1, \mathrm{RH} 2$, and $\mathrm{RH} 3$ are highlighted in 3 schematics: a) Domain architecture with the predicted secondary structure at the location of each deletion. $\mathrm{RH} 1$ is located in the A'a helix of the LOV domain, $\mathrm{RH} 2$ in the linker between the LOV domain and $\alpha 1$ of the $\mathrm{DHp}$ domain, and $\mathrm{RH} 3$ is in the ATP lid. b) Monomeric homology model of RH376 based on the structure of EL346 (Rivera-Cancel et al., 2014). c) Dimeric homology model of RH376 based on the engineered YF1 sensor kinase (Diensthuber et al., 2013). All LOV domains are colored blue, DHp domains green, and CA domains red. Mutations are highlighted and circled. 
With the possibility of monomer-dimer transition as a regulatory mechanism, we sought to isolate the determinants of dimerization in this family of sensor HKs. During evolution, many factors (e.g. changes to protein sequence) can influence the multimeric arrangement of proteins belonging to the same family (Perica et al., 2012; Pillai et al., 2020). To investigate such determinants of the oligomeric state, we compared monomeric EL346 with selected dimeric HKs from all three clades using sequence alignment. These pairwise alignments highlighted four regions of difference in the primary sequence between EL346 and the other HKs; these regions were distributed differently throughout the three clades. However, the dimeric $\mathrm{RH} 376$ from the EL346-like clade contained all four regions of insertions of amino acids between the two proteins (Fig. S3, 5a). We named the regions $\mathrm{RH} 1-4$ and set out to test the hypothesis that one or more of these might be determinants of oligomeric state. Region $\mathrm{RH} 1$ is an insertion of 5 amino acids in RH376 compared to EL346, likely in the A' $\alpha$ helix of the LOV domain. Region $\mathrm{RH} 2$ is an insertion of 7 amino acids in RH376 compared to EL346, located in the linker between the LOV domain and $\alpha 1$ of the $\mathrm{DHp}$ domain. These residues may be in the unstructured linker or form part of an elongated $\alpha 1$ helix; secondary structure prediction by JPred (Drozdetskiy et al., 2015) suggests that these residues are helical (Fig. S3). Region RH3 consists of two insertions, the largest by far, of 19 and 11 amino acids in RH376 compared to EL346, in the ATP lid region. This insert region is predicted to have some helical structure (Fig. S3), unlike the ATP lid of EL346. Region RH4, located at the very C-terminus, is the only unique insertion in EL346, of 10 residues. Coincidentally, RH4 corresponds almost entirely to the highly dynamic C-terminal residues we deleted in an NMR construct of EL346, removal of which had no effect on the monomeric state of that protein (Dikiy et al., 2019). Thus, we did not pursue $\mathrm{RH} 4$ any further as a driver of monomer/dimer state. for LOV domains (RH1, A' $\alpha$ helix) (Banerjee et al., 2016; Diensthuber et al., 2013) and HKs (RH2, DHp domain) (Qin et al., 2000; Tomomori et al., 1999), or large insertions with predicted secondary structure elements $(\mathrm{RH} 3)$, we hypothesized that they could contribute to the

322 dimerization of $\mathrm{RH} 376$ and perhaps be important for dimerization in other HKs. A homology 323 model of the RH376 sequence based on the engineered dimeric LOV-HK YF1 (Diensthuber et 324 al., 2013) illustrates the potential involvement of these residues in dimerization interfaces (Fig.

325 5c). We thus deleted each of the $\mathrm{RH} 1, \mathrm{RH} 2$, and $\mathrm{RH} 3$ regions from the $\mathrm{RH} 376$ sequence,

326 expressed the proteins, and compared them to RH376 WT in terms of LOV photocycle,

327 oligomeric state, and autophosphorylation activity. 

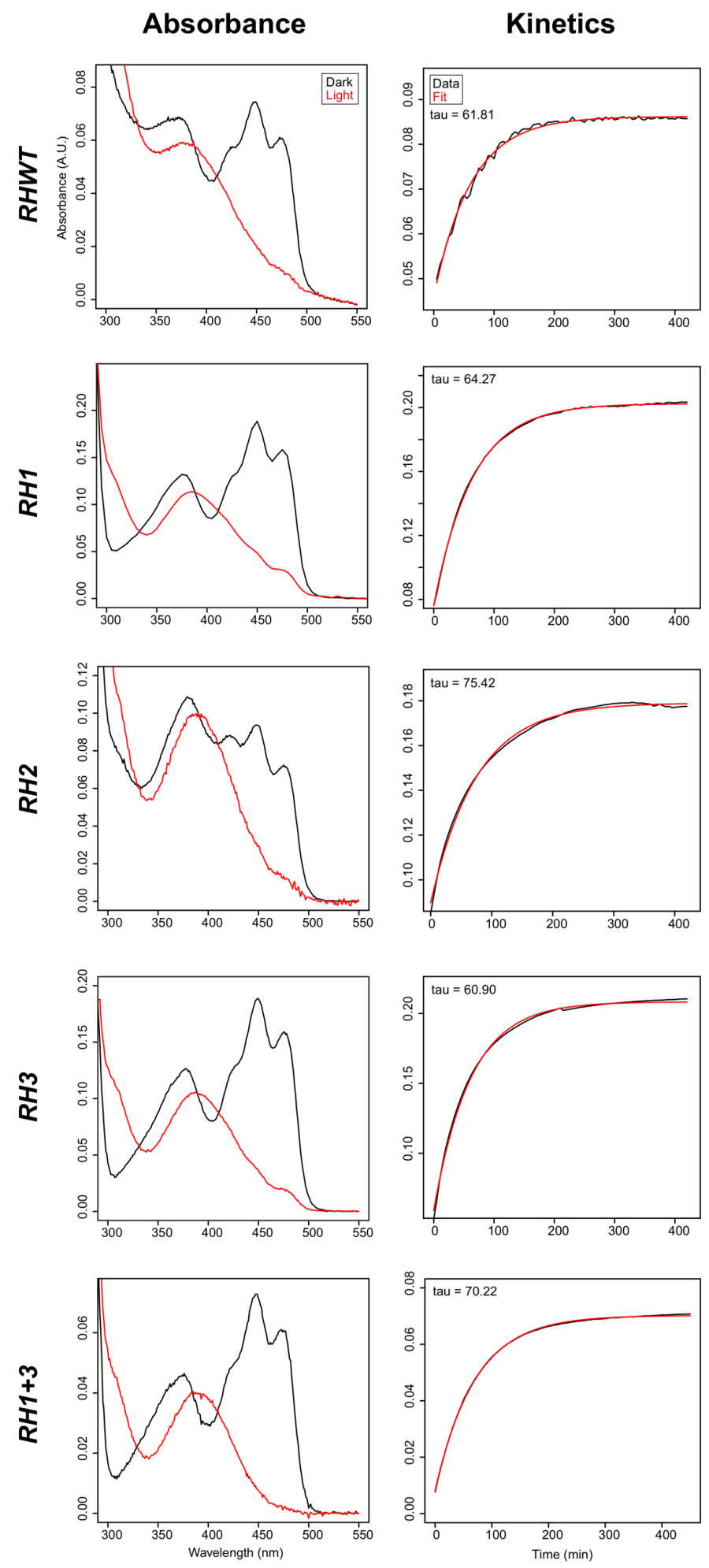

Figure S4: RH376 mutant UV/visible absorbance and dark state reversion kinetics show that mutants are light sensitive with recovery times comparable to the WT (Supplemental to Fig. 6). a) Absorbance of dark and light states along with b) dark state reversion kinetics show that RH376 WT and mutants are light sensitive. Based on tau values extracted from a single exponential decay fit, the photocycle of these mutants is comparable to the WT. 

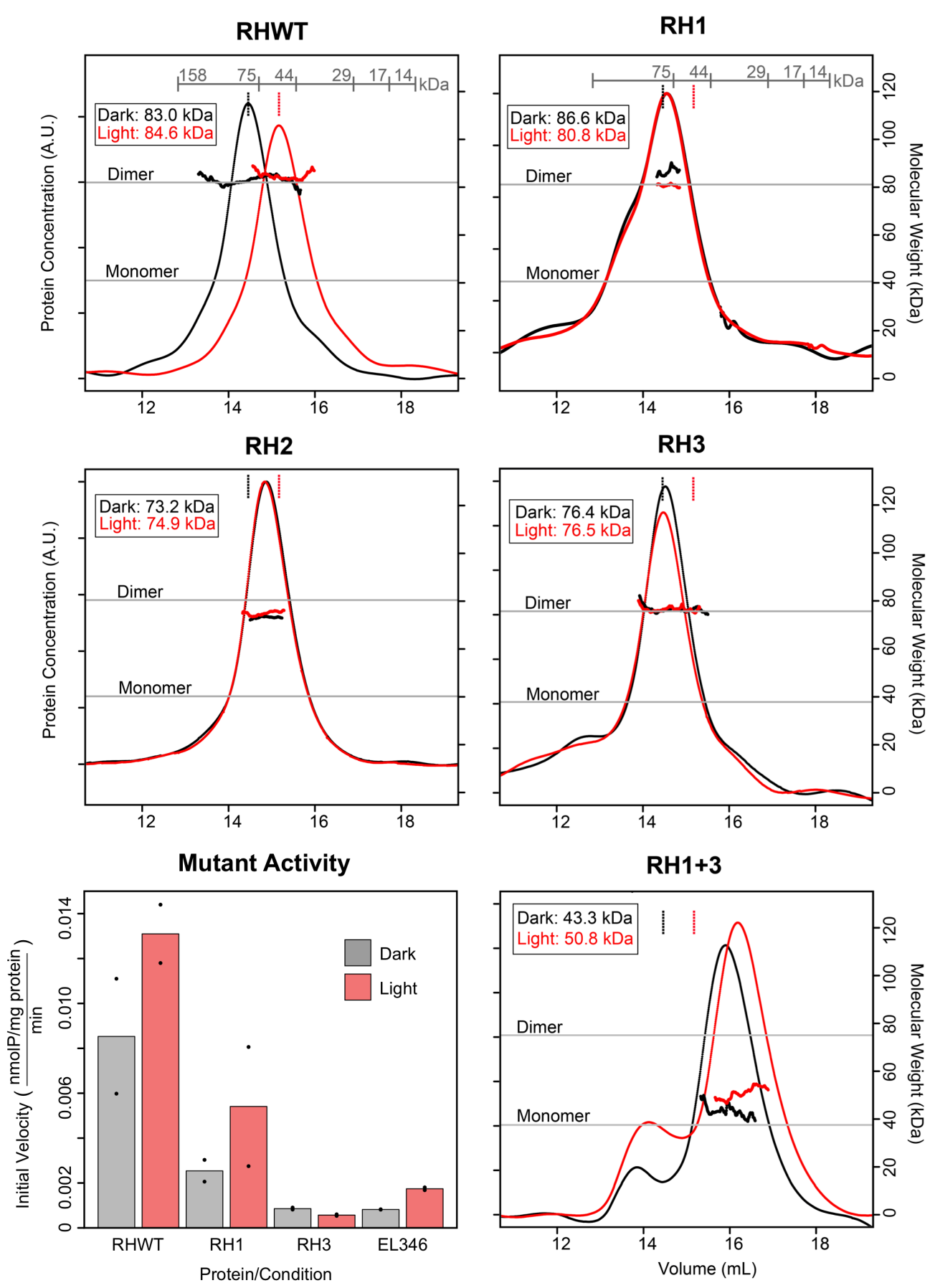

Figure 6: RH376 mutants RH1 and RH3 are active dimers, while double mutant RH1+3 is an inactive monomer. SEC-MALS results show that RH376 WT, RH1 and $\mathrm{RH} 3$ are clear dimers, while $\mathrm{RH} 1+3$ is primarily monomeric. The mass calculated for $\mathrm{RH} 2$ falls between a monomer and dimer, suggesting that the protein exists as a monomer/dimer mixture. The monomeric $\mathrm{RH} 1+3$ exhibits a light-dependent shift in elution peak similar to RHWT that is not present in RH1, RH2 and RH3.

Expected MWs were calculated based on protein sequence. Black and red hash marks represent the position of RH376 WT D and L peaks. Mutant activity, represented by initial velocity calculated from the linear part of the curve in two trials (trials, dots; average, bars), is shown in the bottom left panel, with EL346 values for reference. No activity was detected for RH2 or RH1+3. Light activation can be seen in RH376 WT and RH1, but not in RH3. 
$\mathrm{RH} 1, \mathrm{RH} 2$, and $\mathrm{RH} 3$ proteins display the characteristic LOV absorbance triplet centered at $450 \mathrm{~nm}$ and thermal reversion to the dark state with time constants comparable to that of the WT protein (Fig. S4), suggesting that the deletions do not perturb the folding and function of the LOV domain. In $\mathrm{RH} 2$, we detected a small change in the relative heights of the triplet peaks, indicating that flavin binding is likely adversely affected (Fig. S4) despite the deletion and flavin binding occurring in separate domains (DHp, LOV). We determined the oligomeric state of each deletion mutant by SEC-MALS and found that none of the single deletion constructs elutes as a monomer regardless of dark or light conditions (Fig. 6). However, in each case, the apparent light-induced conformational shift seen in the WT protein is lost. In addition, the measured mass for $\mathrm{RH} 2$ is lower than that expected for a full dimer, suggesting, along with the changed elution volume, that the protein samples both dimeric and monomeric conformations.

\begin{tabular}{|c|c|c|c|c|c|c|}
\hline \multirow{2}{*}{ Protein } & \multirow{2}{*}{ Deletion } & \multirow{2}{*}{$\begin{array}{c}\text { Oligomeric } \\
\text { State }\end{array}$} & \multirow{2}{*}{$\begin{array}{l}\text { Tau } \\
\text { (min) }\end{array}$} & \multicolumn{3}{|c|}{ Initial Velocity $\frac{\mathrm{nmol} \mathrm{PO}_{4}}{\mathrm{mg} \text { protein }} \min ^{-1}$} \\
\hline & & & & Dark & Light & Light:Dark \\
\hline RHWT & $\mathrm{N} / \mathrm{A}$ & Dimer & 61.8 & $\begin{array}{c}8.53 E-03 \pm \\
59.9 \%\end{array}$ & $\begin{array}{c}1.31 \mathrm{E}-02 \pm \\
19.8 \%\end{array}$ & 1.54 \\
\hline RH1 & $A^{\prime} \alpha$ & Dimer & 64.3 & $\begin{array}{c}2.54 \mathrm{E}-03 \pm \\
37.8 \%\end{array}$ & $\begin{array}{c}5.41 \mathrm{E}-03 \pm \\
98.4 \%\end{array}$ & 2.13 \\
\hline RH2 & $\begin{array}{l}\text { LOV/DHp } \\
\text { linker }\end{array}$ & $\begin{array}{c}\text { Monomer/ } \\
\text { Dimer }\end{array}$ & 75.4 & n.d. & n.d. & N/A \\
\hline RH3 & ATP lid & Dimer & 60.9 & $\begin{array}{c}8.57 E-04 \pm \\
12.1 \%\end{array}$ & $\begin{array}{c}5.71 \mathrm{E}-04 \pm \\
11.6 \%\end{array}$ & 0.67 \\
\hline RH1+3 & $\begin{array}{l}\text { A'a and } \\
\text { ATP lid }\end{array}$ & Monomer & 70.2 & n.d. & n.d. & N/A \\
\hline
\end{tabular}

Table S1: RH376 Deletion Mutant Measurements (Supplemental to Fig. 6). Oligomeric states were determined by SEC-MALS. Tau represents the time constant of the thermal reversion of the light state as fit to a first-order exponential decay (Fig. S4). Initial velocities were calculated from the linear region of autophosphorylation activity curves. Error is represented by the percent bias between trials of the same conditions. The ratio of light to dark initial velocities represents the degree of light activation that each protein undergoes.

We suspected that these deletions would not have a major impact on

343 autophosphorylation activity of RH376, given their modest effects on photocycle and

344 oligomerization state. As a baseline, we found that the RH376 WT protein displays robust

345 autophosphorylation activity in the dark, comparable to that of RE356, as well as a $\sim 1.5 \mathrm{x}$

346 increase in initial velocity upon illumination (Fig. 6). The RH1 mutant also shows

347 autophosphorylation activity, although lower than that of the WT protein, with an initial velocity in

348 the dark of about $0.3 x$ of the WT. However, there is still an increase in the initial velocity of 
autophosphorylation in the light. By contrast, $\mathrm{RH} 2$ shows no detectable autophosphorylation in the dark or light (Table S1, Fig. S5), while RH3 shows detectable but severely attenuated activity in the dark ( $0.08 \mathrm{x}$ of the WT initial velocity) with no light-dependent increase in activity (Fig. 6). By comparison, EL346 shows autophosphorylation activity dramatically lower than that of $\mathrm{RH} 376$ WT, on the same order as that of $\mathrm{RH} 3$, but with a detectable light-mediated increase of $\sim 2 x$ (Fig. 6). In the case of $\mathrm{RH} 376$, both the $\mathrm{RH} 2$ and $\mathrm{RH} 3$ variants dramatically decrease

355 autophosphorylation activity despite having different effects on dimerization.

\section{RHWT}

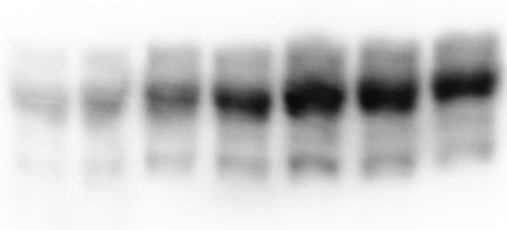

EL346

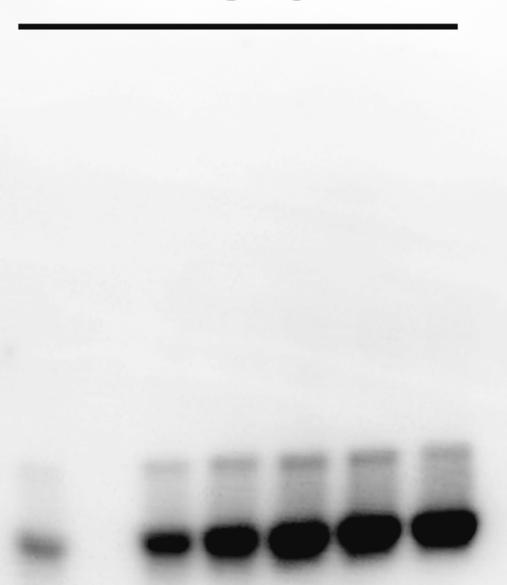

\section{RH2}

Figure S5: RH2 and RH1+3 display no detectible autophosphorylation activity (Supplemental to Fig. 6). The phosphor image of $\mathrm{RH} 2$ and $\mathrm{RH} 1+3$ autophosphorylation assay gels, showing no detectable signal for each of the proteins. Proteins EL346 and RH376 WT were run on the same gel and are shown here for reference. 
would be a synergistic effect. We characterized the LOV absorbance spectrum and photocycle of the so-called $\mathrm{RH} 1+3$ (Fig. S4) and saw no large perturbations. By contrast, SEC-MALS showed a dramatic change in oligomeric state, with $\mathrm{RH} 1+3$ existing mainly as a monomer (Fig. 6), though neither deletion alone showed monomeric character. Interestingly, this monomeric protein also retains the apparent compaction of the protein upon illumination, which was not seen for either $\mathrm{RH} 1$ or $\mathrm{RH} 3$ separately. Additionally, we detected no autophosphorylation activity in $\mathrm{RH} 1+3$ at conditions similar to the other proteins tested (Table S1, Fig. S5). Thus, loss of activity seems to be correlated with $\mathrm{RH} 2$ and $\mathrm{RH} 3$ deletions, with loss of $\mathrm{RH} 3$ region driving a loss of activity in combined mutants as well.

Our data suggest that neither of the segments targeted by $\mathrm{RH} 1$ and $\mathrm{RH} 3$ are sufficient for dimerization, as detected by SEC-MALS. However, they are apparently both necessary, as loss of both regions results in a primarily monomeric protein. For autokinase activity, all regions contribute to activity, with the order of $\mathrm{RH} 1<\mathrm{RH} 3<\mathrm{RH} 2$ in terms of increasing necessity for activity. Thus, activity and dimeric state are not entirely directly correlated, with the dimeric RH3 deletion lacking activity and light-activation as well as the monomeric $\mathrm{RH} 1+3$.

Discussion

Prior to this study, the vast majority of characterized sensor HKs were known to be dimeric; we are aware of only the monomeric LOV-containing EL346 and EL362 from E. litoralis HTCC2594 (Correa et al., 2013; Rivera-Cancel et al., 2014) as exceptions. Our work further expands the behavior of these enzymes to include two proteins in a slow monomer:dimer equilibrium along with three obligate dimers which exhibit a range of large-scale conformational changes upon activation. Our approach to identify these novel sensor HKs relied on sequence homology searches to find homologs of EL346, revealing a large family of related LOV- and PAS-HKs in the Alphaproteobacteria. Most of these proteins were Pfam (El-Gebali et al., 2019) HisKA_2 or HWE-HK-type histidine kinases, which have not been thoroughly characterized structurally (Herrou et al., 2017; Rinaldi et al., 2016; Rivera-Cancel et al., 2014), and we hypothesized that a subset of these sequences with the highest homology to monomeric EL346 might contain other monomers. 

which also cluster within that second clade. SEC-MALS. While ME354 shows minimal change upon illumination, both RT349 and RH376 exhibit light-dependent shifts towards later elution volumes, suggesting that the proteins adopt more compact structures in the light while not changing the apparent MW of either protein. Notably, RT349 in the light appears to consist of two overlapped peaks, indicating multiple conformations, one of which corresponds to a globular dimer and the other to an expanded monomer state (Fig. S6); in contrast, RH376 appears to shift to a single, compacted structure. These shifts appear to be more substantial than the light-dependent motions reported in the activation of either the engineered dimeric YF1 or natural monomeric EL346 LOV-HKs (Berntsson et al., 2017; Diensthuber et al., 2013; Dikiy et al., 2019; Rivera-Cancel et al., 2014). 
how they relate to the large conformational changes postulated to accompany activation from crystal structures of inactive HKs (Casino et al., 2014; Mechaly et al., 2014).

Turning to the two PAS-HKs, both RE356 and MS367 interconvert slowly enough between dimeric and monomeric states that we could separate them by size exclusion

412 chromatography. The stable PAS-HK MS367 monomer is also active in autophosphorylation,

413 albeit at a lower rate than the dimer, suggesting that shifts in the monomer/dimer equilibrium

414 could regulate sensor HK enzymatic activity, as has been demonstrated or postulated for a

415 range of signaling proteins including certain sensor HKs (Lee et al., 2008), receptor tyrosine

416 kinases (Yarden \& Schlessinger, 1987), and photoreceptors (Liao et al., 2020; Sang et al.,

417 2005; Zoltowski \& Crane, 2008; Zoltowski et al., 2013). Dimerization may be modulated by

418 potential ligand binding in the yet-uncharacterized PAS domains of these HKs, as in other PAS-

419 domain containing signaling proteins (Scheuermann et al., 2009), or via other mechanisms. We

420 also suggest that the relative instability of the dimer state, which forms higher-molecular-weight

421 aggregates over time, may serve as an intrinsic timer on the more active signaling species to

422 temporally limit a biological response post-activation.

423 Given the integral role of dimerization in this possible regulation, along with its obvious

424 necessity in trans-phosphorylation (Ashenberg et al., 2013) within HKs, we sought to

425 understand the sequence determinants of dimerization with an engineering approach. Using

426 RH376 as a model dimer, we systematically removed combinations of the three insertions it

427 contains compared to the monomeric EL346 (Figs 5 and S3), hypothesizing that one or more of

428 these changes could create a monomeric LOV-HK. While each deletion mutant was well-folded

429 as evidenced by binding flavin chromophores and undergoing canonical LOV photochemistry,

430 we found that all three single deletion mutants $(\mathrm{RH} 1, \mathrm{RH} 2, \mathrm{RH} 3)$ remained largely dimeric

431 despite the removal of 5-30 amino acid residues apiece. Each, however, lost the light-induced

432 compaction seen in SEC-MALS analysis of the wildtype protein. Remarkably, the combination of

433 two of these deletions to generate the $\mathrm{RH} 1+3$ construct both monomerized the protein and

434 restored the light-induced conformational shift seen in the WT parent sequence. From these

435 studies, we arrive at two general conclusions: 1) multiple regions determine oligomeric states in

436 this family of sensor HKs, and 2) light-induced conformational changes have a complex

437 relationship with autophosphorylation activity and do not appear to be restricted to dimeric or

438 monomeric states.

439 The first of these conclusions, the use of distributed interfaces for dimerization, is 440 consistent with structural studies on the dimeric YF1 engineered sensor kinase (Diensthuber et 441 al., 2013) showing intermonomer contacts via residues in both the LOV domain A' $\alpha$ helix and 
442 DHp domain, which are typical dimerization interfaces for LOV photosensors (Diensthuber et al.,

443 2013; Key et al., 2007) and canonical HisKA histidine kinases (Ashenberg et al., 2013; Dutta et

444 al., 1999) respectively. These correspond to our $\mathrm{RH} 1$ and $\mathrm{RH} 2$ deletions; neither of these

445 changes, nor the RH3 deletion in a large loop near the kinase ATP binding site, were sufficient

446 on its own to generate monomers, but each had markedly different effects on

447 autophosphorylation. These data suggest different types of dimers may be generated by these

448 deletions, some of which retain function better than others. Of surprise to us, the RH3 deletion -

449 which removes a large loop near the ATP binding site - affected dimerization when paired with

$450 \mathrm{RH} 1$. While this site has not been previously described as being involved in dimerization to the

451 best of our knowledge, we note that ATP itself bridges interactions between multiple domains in

452 both known HWE/HisKA2 HK crystal structures (Rinaldi et al., 2016; Rivera-Cancel et al., 2014),

453 laying some precedent for interactions at or near the nucleotide to be involved in dimerization.

454 Clearly, further structural work is needed to more fully assess how dimerization is encoded

455 among multiple HKs.

456 In addition, our work underscores the diversity of structural changes which occur as part

457 of the functional activation process among HKs, another long-discussed topic within the

458 community. Considering the low-resolution conformational changes we observe by SEC-MALS

459 upon photoactivation of the LOV-HKs tested here, we see a wide span of responses. It is clear

460 from the detailed structures available among HKs, chiefly on inactive forms, that substantial

461 rearrangements must be required to let catalytic domains transit long distances to

462 phosphorylate substrate histidines within DHp domains (Diensthuber et al., 2013; Marina et al.,

463 2005; Rivera-Cancel et al., 2014). Solution integrated structural biology approaches are

464 beginning to reveal such changes with fewer experimental caveats than crystallographic

465 approaches (Berntsson et al., 2017; Dikiy et al., 2019; Ghosh et al., 2019) but this essential

466 process still remains undercharacterized at present.

467 The diversity of oligomeric states in a family of sensor HKs that we uncover here opens

468 the door to novel regulatory modes within this family, such as control of dimerization, whether by

469 ligand or inherent thermodynamic stability, or cooperativity. The closely related bacterial

470 chemoreceptor proteins, for instance, form large arrays in order to increase signaling output

471 through cooperativity (Muok et al., 2020). Similar use of monomer/dimer transitions and

472 evolution of variable quaternary structure is at the heart of acquiring cooperativity and novel

473 regulatory modes in the classic hemoglobin family of oxygen-binding proteins, which started as

474 ancestral monomers prior to dimerizing by gene duplication events and the acquisition of a

475 relatively small number of mutations (Pillai et al., 2020). Our findings suggest that different 
476 oligomeric states within the sensor HK family could allow for different potential for cooperativity,

477 perhaps depending on the input signal or output pathway, or integrating multiple points of

478 control or regulation to be added to the canonical signal transduction pathway of two-component

479 systems.

480

\section{Acknowledgements}

482 The authors would like to acknowledge Bright Shi for generating the $\mathrm{RH} 1+3$ construct, as well 483 as Matthew Cleere, Zaynab Jaber, Jaynee Hart, and other Gardner lab members for fruitful 484 discussions. This work was supported by NIH grants R01 GM106239 (to K.H.G.) and F32 485 GM1119311 (to I.D.).

Competing interests

488 The authors declare no competing interests.

\section{References}

491 Ashenberg, O., Keating, A. E., \& Laub, M. T. (2013). Helix bundle loops determine whether histidine kinases autophosphorylate in cis or in trans. J Mol Biol, 425(7), 1198-1209. doi:10.1016/j.jmb.2013.01.011

Banerjee, A., Herman, E., Kottke, T., \& Essen, L. O. (2016). Structure of a Native-like Aureochrome 1a LOV Domain Dimer from Phaeodactylum tricornutum. Structure, 24(1), 171-178. doi:10.1016/j.str.2015.10.022

Berntsson, O., Diensthuber, R. P., Panman, M. R., Bjorling, A., Gustavsson, E., Hoernke, M., . . . Westenhoff, S. (2017). Sequential conformational transitions and alpha-helical supercoiling regulate a sensor histidine kinase. Nat Commun, 8(1), 284. doi:10.1038/s41467-017-00300-5

Bhate, M. P., Molnar, K. S., Goulian, M., \& DeGrado, W. F. (2015). Signal transduction in histidine kinases: insights from new structures. Structure, 23(6), 981-994. doi:10.1016/j.str.2015.04.002

Casino, P., Miguel-Romero, L., \& Marina, A. (2014). Visualizing autophosphorylation in histidine kinases. Nat Commun, 5, 3258. doi:10.1038/ncomms4258

Correa, F., Ko, W. H., Ocasio, V., Bogomolni, R. A., \& Gardner, K. H. (2013). Blue light regulated two-component systems: enzymatic and functional analyses of light-oxygenvoltage (LOV)-histidine kinases and downstream response regulators. Biochemistry, 52(27), 4656-4666. doi:10.1021/bi400617y 
Crosson, S., \& Moffat, K. (2001). Structure of a flavin-binding plant photoreceptor domain: insights into light-mediated signal transduction. Proc Natl Acad Sci U S A, 98(6), 29953000. doi: $10.1073 /$ pnas. 051520298

Crosson, S., Rajagopal, S., \& Moffat, K. (2003). The LOV domain family: photoresponsive signaling modules coupled to diverse output domains. Biochemistry, 42(1), 2-10. doi:10.1021/bi026978I

Diensthuber, R. P., Bommer, M., Gleichmann, T., \& Moglich, A. (2013). Full-length structure of a sensor histidine kinase pinpoints coaxial coiled coils as signal transducers and modulators. Structure, 21(7), 1127-1136. doi:10.1016/j.str.2013.04.024

Dikiy, I., Edupuganti, U. R., Abzalimov, R. R., Borbat, P. P., Srivastava, M., Freed, J. H., \& Gardner, K. H. (2019). Insights into histidine kinase activation mechanisms from the monomeric blue light sensor EL346. Proc Natl Acad Sci U S A, 116(11), 4963-4972. doi:10.1073/pnas. 1813586116

Drozdetskiy, A., Cole, C., Procter, J., \& Barton, G. J. (2015). JPred4: a protein secondary structure prediction server. Nucleic Acids Res, 43(W1), W389-394. doi:10.1093/nar/gkv332

Dutta, R., Qin, L., \& Inouye, M. (1999). Histidine kinases: diversity of domain organization. Mol Microbiol, 34(4), 633-640. doi:10.1046/j.1365-2958.1999.01646.x

Edgar, R. C. (2004). MUSCLE: multiple sequence alignment with high accuracy and high throughput. Nucleic Acids Res, 32(5), 1792-1797. doi:10.1093/nar/gkh340

El-Gebali, S., Mistry, J., Bateman, A., Eddy, S. R., Luciani, A., Potter, S. C., . . Finn, R. D. (2019). The Pfam protein families database in 2019. Nucleic Acids Res, 47(D1), D427D432. doi:10.1093/nar/gky995

Galperin, M. Y. (2018). What bacteria want. Environ Microbiol, 20(12), 4221-4229. doi:10.1111/1462-2920.14398

Gao, R., \& Stock, A. M. (2009). Biological insights from structures of two-component proteins. Annu Rev Microbiol, 63, 133-154. doi:10.1146/annurev.micro.091208.073214

Gasteiger, E., Hoogland, C., Gattiker, A., Duvaud, S. e., Wilkins, M. R., Appel, R. D., \& Bairoch, A. (2005). Protein Identification and Analysis Tools on the ExPASy Server. In J. M. Walker (Ed.), The Proteomics Protocols Handbook (pp. 571-607). Totowa, NJ: Humana Press.

Ghosh, M., Wang, L. C., Huber, R. G., Gao, Y., Morgan, L. K., Tulsian, N. K., . . Anand, G. S. (2019). Engineering an Osmosensor by Pivotal Histidine Positioning within Disordered Helices. Structure, 27(2), 302-314 e304. doi:10.1016/j.str.2018.10.012 
544

545

546

547

548

549

550

551

552

553

554

555

556

557

558

559

560

561

562

563

564

565

566

567

568

569

570

571

572

573

574

575

576

577

Gushchin, I., \& Gordeliy, V. (2018). Transmembrane Signal Transduction in Two-Component Systems: Piston, Scissoring, or Helical Rotation? BioEssays : news and reviews in molecular, cellular and developmental biology, 40(2). doi:10.1002/bies.201700197

Herrou, J., Crosson, S., \& Fiebig, A. (2017). Structure and function of HWE/HisKA2-family sensor histidine kinases. Curr Opin Microbiol, 36, 47-54. doi:10.1016/j.mib.2017.01.008

Karniol, B., \& Vierstra, R. D. (2004). The HWE histidine kinases, a new family of bacterial twocomponent sensor kinases with potentially diverse roles in environmental signaling. $J$ Bacteriol, 186(2), 445-453. doi:10.1128/jb.186.2.445-453.2004

Kenney, L. J., \& Anand, G. S. (2020). EnvZ/OmpR Two-Component Signaling: An Archetype System That Can Function Noncanonically. EcoSal Plus, 9(1). doi:10.1128/ecosalplus.ESP-0001-2019

Key, J., Hefti, M., Purcell, E. B., \& Moffat, K. (2007). Structure of the redox sensor domain of Azotobacter vinelandii NifL at atomic resolution: signaling, dimerization, and mechanism. Biochemistry, 46(12), 3614-3623. doi:10.1021/bi0620407

Lee, J., Tomchick, D. R., Brautigam, C. A., Machius, M., Kort, R., Hellingwerf, K. J., \& Gardner, K. H. (2008). Changes at the KinA PAS-A dimerization interface influence histidine kinase function. Biochemistry, 47(13), 4051-4064. doi:10.1021/bi7021156

Letunic, I., \& Bork, P. (2019). Interactive Tree Of Life (iTOL) v4: recent updates and new developments. Nucleic Acids Res, 47(W1), W256-W259. doi:10.1093/nar/gkz239

Liao, X., Liu, W., Yang, H. Q., \& Jenkins, G. I. (2020). A dynamic model of UVR8 photoreceptor signalling in UV-B-acclimated Arabidopsis. New Phytol, 227(3), 857-866. doi:10.1111/nph.16581

Luo, B. H., Carman, C. V., \& Springer, T. A. (2007). Structural basis of integrin regulation and signaling. Annu Rev Immunol, 25, 619-647. doi:10.1146/annurev.immunol.25.022106.141618

Marina, A., Waldburger, C. D., \& Hendrickson, W. A. (2005). Structure of the entire cytoplasmic portion of a sensor histidine-kinase protein. Embo j, 24(24), 4247-4259. doi:10.1038/sj.emboj.7600886

Mechaly, A. E., Sassoon, N., Betton, J. M., \& Alzari, P. M. (2014). Segmental helical motions and dynamical asymmetry modulate histidine kinase autophosphorylation. PLOS Biol, 12(1), e1001776. doi:10.1371/journal.pbio.1001776

Mechaly, A. E., Soto Diaz, S., Sassoon, N., Buschiazzo, A., Betton, J. M., \& Alzari, P. M. (2017). Structural Coupling between Autokinase and Phosphotransferase Reactions in a Bacterial Histidine Kinase. Structure, 25(6), 939-944 e933. doi:10.1016/j.str.2017.04.011 
Möglich, A. (2019). Signal transduction in photoreceptor histidine kinases. Protein Sci, 28(11), 1923-1946. doi:10.1002/pro.3705

Muok, A. R., Briegel, A., \& Crane, B. R. (2020). Regulation of the chemotaxis histidine kinase CheA: A structural perspective. Biochim Biophys Acta Biomembr, 1862(1), 183030. doi:10.1016/j.bbamem.2019.183030

Perica, T., Chothia, C., \& Teichmann, S. A. (2012). Evolution of oligomeric state through geometric coupling of protein interfaces. Proc Natl Acad Sci U S A, 109(21), 8127-8132. doi:10.1073/pnas.1120028109

Pillai, A. S., Chandler, S. A., Liu, Y., Signore, A. V., Cortez-Romero, C. R., Benesch, J. L. P., . . . Thornton, J. W. (2020). Origin of complexity in haemoglobin evolution. Nature, 581(7809), 480-485. doi:10.1038/s41586-020-2292-y

Qin, L., Dutta, R., Kurokawa, H., Ikura, M., \& Inouye, M. (2000). A monomeric histidine kinase derived from EnvZ, an Escherichia coli osmosensor. Mol Microbiol, 36(1), 24-32. doi:10.1046/j.1365-2958.2000.01837.x

Rinaldi, J., Arrar, M., Sycz, G., Cerutti, M. L., Berguer, P. M., Paris, G., . . Goldbaum, F. A. (2016). Structural Insights into the HWE Histidine Kinase Family: The Brucella Blue Light-Activated Histidine Kinase Domain. J Mol Biol, 428(6), 1165-1179.

doi:10.1016/j.jmb.2016.01.026

Pudasaini, A., El-Arab, K. K., \& Zoltowski, B. D. (2015). LOV-based optogenetic devices: lightdriven modules to impart photoregulated control of cellular signaling. Front Mol Biosci, 2, 18. doi:10.3389/fmolb.2015.00018

Purcell, E. B., McDonald, C. A., Palfey, B. A., \& Crosson, S. (2010). An analysis of the solution structure and signaling mechanism of LovK, a sensor histidine kinase integrating light and redox signals. Biochemistry, 49(31), 6761-6770. doi:10.1021/bi1006404

Rivera-Cancel, G., Ko, W. H., Tomchick, D. R., Correa, F., \& Gardner, K. H. (2014). Full-length structure of a monomeric histidine kinase reveals basis for sensory regulation. Proc Natl Acad Sci U S A, 111(50), 17839-17844. doi:10.1073/pnas.1413983111

Rueden, C. T., Schindelin, J., Hiner, M. C., DeZonia, B. E., Walter, A. E., Arena, E. T., \& Eliceiri, K. W. (2017). ImageJ2: ImageJ for the next generation of scientific image data. BMC Bioinformatics, 18(1), 529. doi:10.1186/s12859-017-1934-z

Sang, Y., Li, Q. H., Rubio, V., Zhang, Y. C., Mao, J., Deng, X. W., \& Yang, H. Q. (2005). Nterminal domain-mediated homodimerization is required for photoreceptor activity of Arabidopsis CRYPTOCHROME 1. Plant Cell, 17(5), 1569-1584. doi:10.1105/tpc.104.029645 
612 Scheuermann, T. H., Tomchick, D. R., Machius, M., Guo, Y., Bruick, R. K., \& Gardner, K. H.

613

614

615

616

617

618

619

620

621

622

623

624

625

626

627

628

629

630

631

632

633

634

635

636

637

638

639

640

641

642

643

644

645

(2009). Artificial ligand binding within the HIF2alpha PAS-B domain of the HIF2 transcription factor. Proc Natl Acad Sci U S A, 106(2), 450-455.

doi:10.1073/pnas.0808092106

Schindelin, J., Arganda-Carreras, I., Frise, E., Kaynig, V., Longair, M., Pietzsch, T., . . . Cardona, A. (2012). Fiji: an open-source platform for biological-image analysis. Nat Methods, 9(7), 676-682. doi:10.1038/nmeth.2019

Sheffield, P., Garrard, S., \& Derewenda, Z. (1999). Overcoming expression and purification problems of RhoGDI using a family of "parallel" expression vectors. Protein Expr Purif, 15(1), 34-39. doi:10.1006/prep.1998.1003

Sievers, F., Wilm, A., Dineen, D., Gibson, T. J., Karplus, K., Li, W., . . Higgins, D. G. (2011). Fast, scalable generation of high-quality protein multiple sequence alignments using Clustal Omega. Molecular systems biology, 7, 539. doi:10.1038/msb.2011.75

Staroń, A., \& Mascher, T. (2010). General stress response in alpha-proteobacteria: PhyR and beyond. Mol Microbiol, 78(2), 271-277. doi:10.1111/j.1365-2958.2010.07336.x

Swartz, T. E., Tseng, T. S., Frederickson, M. A., Paris, G., Comerci, D. J., Rajashekara, G., . . . Bogomolni, R. A. (2007). Blue-light-activated histidine kinases: two-component sensors in bacteria. Science, 317(5841), 1090-1093. doi:10.1126/science.1144306

Szurmant, H., White, R. A., \& Hoch, J. A. (2007). Sensor complexes regulating two-component signal transduction. Curr Opin Struct Biol, 17(6), 706-715. doi:10.1016/j.sbi.2007.08.019

Tomomori, C., Tanaka, T., Dutta, R., Park, H., Saha, S. K., Zhu, Y., . . I Ikura, M. (1999). Solution structure of the homodimeric core domain of Escherichia coli histidine kinase EnvZ. Nat Struct Biol, 6(8), 729-734. doi:10.1038/11495

Tseng, T. S., Frederickson, M. A., Briggs, W. R., \& Bogomolni, R. A. (2010). Light-activated bacterial LOV-domain histidine kinases. Methods Enzymol, 471, 125-134. doi:10.1016/S0076-6879(10)71008-9

Wang, S. (2012). Bacterial Two-Component Systems: Structures and Signaling Mechanisms. In C. Huang (Ed.), Protein Phosphorylation in Human Health: InTech.

West, A. H., \& Stock, A. M. (2001). Histidine kinases and response regulator proteins in twocomponent signaling systems. Trends Biochem Sci, 26(6), 369-376. doi:10.1016/s09680004(01)01852-7

Yarden, Y., \& Schlessinger, J. (1987). Epidermal growth factor induces rapid, reversible aggregation of the purified epidermal growth factor receptor. Biochemistry, 26(5), 14431451. doi:10.1021/bi00379a035 
646 Zoltowski, B. D., \& Crane, B. R. (2008). Light activation of the LOV protein vivid generates a

647 rapidly exchanging dimer. Biochemistry, 47(27), 7012-7019. doi:10.1021/bi8007017

648 Zoltowski, B. D., \& Gardner, K. H. (2011). Tripping the light fantastic: blue-light photoreceptors

649 as examples of environmentally modulated protein-protein interactions. Biochemistry,

$650 \quad 50(1), 4-16$. doi:10.1021/bi101665s

651 Zoltowski, B. D., Motta-Mena, L. B., \& Gardner, K. H. (2013). Blue light-induced dimerization of

652 a bacterial LOV-HTH DNA-binding protein. Biochemistry, 52(38), 6653-6661.

653 doi:10.1021/bi401040m

654 